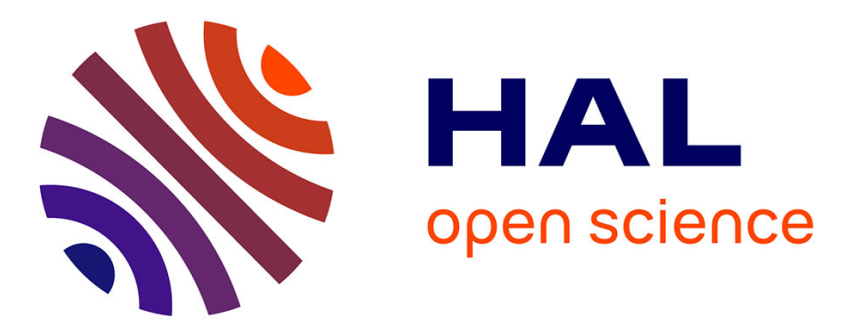

\title{
Cost over Progress Based Energy Efficient Routing over Virtual Coordinates in Wireless Sensor Networks
}

\author{
Essia Hamouda Elhafsi, Nathalie Mitton, David Simplot-Ryl
}

\section{To cite this version:}

Essia Hamouda Elhafsi, Nathalie Mitton, David Simplot-Ryl. Cost over Progress Based Energy Efficient Routing over Virtual Coordinates in Wireless Sensor Networks. IEEE International Workshop "From Theory To Practice in Wireless Sensor Networks" (t2pWSN), Jun 2007, Helsinki, Finland. pp.1-6. hal-00383999

\section{HAL Id: hal-00383999 \\ https://hal.science/hal-00383999}

Submitted on 14 May 2009

HAL is a multi-disciplinary open access archive for the deposit and dissemination of scientific research documents, whether they are published or not. The documents may come from teaching and research institutions in France or abroad, or from public or private research centers.
L'archive ouverte pluridisciplinaire HAL, est destinée au dépôt et à la diffusion de documents scientifiques de niveau recherche, publiés ou non, émanant des établissements d'enseignement et de recherche français ou étrangers, des laboratoires publics ou privés. 


\title{
Cost over Progress Based Energy Efficient Routing over Virtual Coordinates in Wireless Sensor Networks
}

\author{
Essia H. Elhafsi ${ }^{\dagger}$, Nathalie Mitton ${ }^{\ddagger}$ and David Simplot-Ryl ${ }^{\ddagger}$ \\ IRCICA/LIFL, Univ. Lille 1, CNRS UMR 8022, INRIA Futurs, France \\ †essia@cs.ucr.edu, ${ }^{\ddagger}\{$ mitton, simplot-Ryl $\} @$ lifl.fr
}

\begin{abstract}
We propose an energy efficient routing protocol, VCost, for sensor networks. We assume that nodes are unaware of their geographic location thus, VCost assigns virtual coordinates to nodes as follows. Based on the node hop count distances from a set of landmarks, our method computes a distance metric to obtain the node's virtual coordinates. $V C o s t$, then uses these coordinates to route packets from node $u$ to node $v$, in its neighborhood, such that the ratio of the cost to send a message to $v$ to the progress in the routing task towards the destination is minimized. Compared to existing algorithms that use virtual locations, our simulation shows that VCost improves significantly energy consumption and preserves the small percentage of successful routings.
\end{abstract}

\section{Introduction}

Sensor networks are specialized ad hoc networks composed of a large number of self organizing devices. They are used in a wide range of applications, such as monitoring, security, and data-gathering. These applications have two challenging issues in common i. e., energy savings and position-awareness. In this paper, we address these two key issues to efficiently deploy sensor networks.

Nodes, in sensor networks, rely on batteries with limited capacity, thus the most important criteria when designing communication protocols is to optimize their energy consumption to extend the life of the sensor device and extend the reliability of the underlying network. In this framework, routing protocols based on geographic information of the sensors have been proposed as a viable alternative to existing routing protocols for wireless ad hoc networks in order to reduce the overhead of maintaining routing tables in the sensors and to avoid the cost (energy consumption) of flooding and route discovery. However, such a solution requires that the sensors be aware of their geographic (physical) position which can be obtained by equipping all the sensors with costly global positioning system (GPS) devices. An approximate solution may be obtained by equipping only a few designated sensors (called landmarks) with GPS devices and let the remaining nodes infer their positions. However, even such an expensive alternative may not be a reliable solution since GPS reception might be obstructed by static obstacles $i$. e., nodes may be deployed indoors. A cheaper alternative is to consider the problem of inferring the locations of the nodes in sensor networks in which no node is aware of its physical position. Proposed solutions such as in $[1,2,4,6,7]$ are aimed at routing by deriving and using virtual coordinates. However, none of the above cited papers consider or optimize the energy consumption in their proposed algorithms.

In this paper, we are interested in energy efficient routing in sensor networks where nodes are not aware of their physical locations. Xu et al., in [8], compute the optimal transmission radius that minimizes the total power consumption for a routing task in sensor network. In this work, we extend this result and present an energy aware routing algorithm based on virtual coordinates. We compare the performance of our proposed method to the one of several geographic routing algorithms and show that our algorithm is efficient in terms of energy saving and hit rate (success rate of a message to reach its final destination).

The rest of the paper is organized as follows. In Section 2, we present a brief summary of existing geographic routing algorithms relevant to our work. In Section 3, we present our contribution, a cost efficient routing algorithm over virtual coordinates. In Section 4, we compare the performance of our proposed method to alternative routing algorithms presented in Section 2. Finally we conclude and present future work extensions.

\section{Related Works}

A sensor network is generally represented by a graph $G=(V, E)$ where $V$ is the set of nodes and $E \subseteq V^{2}$ 
is the set of edges which allow the available communications. If $u$ and $v$ are two nodes of the graph $G$, then the edge $(u, v)$ belongs to $E$ means that $u$ is physically neighbor of $v$ and thus it receives its messages. We denote by $R$ the maximum transmission range of communication which is the same for all nodes in $G$. Let $\operatorname{dist}(u, v)$ be the Euclidean distance between $u$ and $v$. The set $E$ is then defined as follows: $E=\left\{(u, v) \in V^{2} \mid \operatorname{dist}(u, v) \leq R\right\}$. We define the neighborhood set $N(u)$ of a node $u$ as: $N(u)=\{v \in V \mid v \neq u \wedge(u, v) \in E\}$.

To route a packet from a given source $s$ to a given destination $d$ in $G$, the geographic location of the nodes are generally required. However, several geographic routing approaches that rely on virtual coordinates have been proposed. To define a virtual coordinate system, one of three options can be adopted. First, one can assume that nodes have knowledge of the position of their neighbors and based on this information a coordinate system can be defined. Second, one can equip a subset of nodes with a satellite receiver and use these nodes as landmarks. In this case, to infer the position of the remaining nodes, it suffices to know their distances relative to the landmarks. Several distance measures, such as the Euclidean and the Hamming distances, have been used in the literature. A Virtual Coordinate assignment (VCap) protocol is proposed in [2] as a third option when no location information is available.

In this work, we focus on the following routing algorithms: Most Forward Routing, Gradient landmark based routing, VCap Routing and Cost-over progress Routing.

- Most Forward Routing (MFR). In this greedy approach [9], the source node forwards the message to exactly one of its neighbors that is closest to the destination. This is a simple localized algorithm however that does not guarantee delivery. There are several scenarios in which this greedy routing fails. For instance, if a node $u$ is closer to the destination than any of its neighbors, or if two adjacent nodes are equally close to the destination and none of their neighbors is closer, then this approach can be trapped in a local minimum and the algorithm fails to find a path to the final destination. Thus the hit rate is very low. However, this algorithm works well in a dense graph.

- Gradient landmark based routing (Glider). In [4] nodes are partitioned into tiles and a set of well dispersed nodes are identified as landmarks. Virtual coordinates are then given to each node based on their centered square-distance, otherwise known as the variance, from each landmark. Based on the virtual coordinate system, the distance between two nodes, the centered virtual distance is computed. In our work, we are only interested in Glider's virtual coordinate system, but for completeness we describe its routing scheme. A node first computes a sequence of tiles for the routing path. Then it uses gradient descent on the Euclidean distance function in the virtual coordinates towards the next tile in the path. This scheme does not guarantee delivery in the discrete case. Moreover, it is complex and requires a high communication overhead, $i$. e., storage of a view of the communication graph and node location that should be accessible by all nodes in the network.

- Virtual Coordinate assignment protocol (VCap). In [2] a system of virtual coordinates based on hop counts from three landmarks is proposed. Nodes are assigned a triplet of coordinates given as the number of hops the node is distant from each landmark. Then, nodes use a greedy routing, like $M F R$ with the Hamming distance computed on these coordinates. Note that even though, an efficient coordinate system can be generated using three landmarks, a more accurate one can be established as the number of landmarks increases as we show in this work and as claimed in [1].

- Cost Over Progress routing. In [5], a localized energyaware routing algorithm where nodes are equipped with GPS receivers is proposed. Each node makes a routing decision on the basis of its location, its neighbors and the destination. A node forwards the packet to the neighbor closer to the destination such that the ratio of the energy consumed to the progress made (measured as the reduction in distance to destination) is minimized. Generally, the energy consumed $\mathrm{J}$, depends on the transmission range $r$ and the overhead $c$ that is due to signal processing and it is equal to $\mathrm{J}=r^{\alpha}+c$ if $r \neq 0$ and zero otherwise, $\alpha$ is a real constant greater than 1 and it represents the signal attenuation. In this work, we ignore the energy consumed due to signal emission. In [8] the optimal transmission radius, $r^{*}$, that minimizes the total power consumption for a routing task is computed and it is equal to: $\mathrm{r}^{*}=\sqrt[\alpha]{\frac{\mathrm{c}}{\alpha-1}}$.

\section{Cost over Progress over Virtual Coordinates}

The framework of our proposition is similar to VCap. Several nodes, $L_{1}, \ldots, L_{k}$ with $k \geq 3$, in the network are distinguished as landmarks. An arbitrary node $x$ knows its distance vector $l(x)=\left(l_{1}, \ldots, l_{k}\right)$ where $l_{i}$ is the hopdistance between $x$ and $L_{i}$. From vector $l(x)$, the node generates a so-called virtual coordinates $c(x)=\left(x_{1}, \ldots, x_{m}\right)$ with $m \geq 2$. Note that in general $m \leq k$, in our study $m=k$. This computation function is denoted by $\Gamma$. We consider two $\Gamma$ functions: the identity denoted by $\Gamma_{i d}$ $\left(x_{i}=l_{i}\right)$ and the "centered virtual coordinates" used in [4] 
and denoted by $\Gamma_{c v c}\left(x_{i}=l_{i}^{2}-\mu\right.$ where $\left.\mu=\frac{1}{k} \sum_{i=1}^{k} l_{i}^{2}\right)$. We suppose that each node $x$ knows the virtual coordinates of each node in its neighborhood $(N(x))$.

To route a packet to destination $d$, a node extracts the virtual coordinates of $d$ from the packet and chooses a forwarding node in its neighborhood. We propose to use "cost over progress" presented in [5]. The idea is that the current node $x$ chooses node $y \in N(x)$ which minimizes $\frac{\operatorname{cost}(x, y)}{\operatorname{progress}(x, y, d)}$ where $\operatorname{cost}(x, y)$ represents the "cost" of $x$ to send the message to its neighbor $y$, and where $\operatorname{progress}(x, y, d)$ is the progress in the routing task. Basically, the progress can be expressed as the difference $\operatorname{dist}(x, d)-\operatorname{dist}(y, d)$ where $\operatorname{dist}(u, v)$ is the "distance" between nodes $u$ and node $v$.

For this protocol to work, the current node has to limit its choices to neighbors with positive progress.

In this paper, we consider two cost functions: $\operatorname{cost}_{1}(x, y)=1$ when node $x$ is not able to adapt its communication range and $\operatorname{cost}_{e}(x, y)=|x y|^{\alpha}+c$ otherwise. The distance $|x y|$ is the geographic distance between node $x$ and node $y$.

For the distance function dist, we consider three different functions. The first one, called "Hamming distance", defined by $\operatorname{dist}_{h}(x, y)=\sum_{i=1}^{m}\left|x_{i}-y_{i}\right|$. The second function, called "Euclidean distance", defined by $\operatorname{dist}_{e}(x, y)=$ $\sqrt{\sum_{i=1}^{m}\left(x_{i}-y_{i}\right)^{2}}$. The last distance function is called "Square Euclidean" distance and it is simply defined by $\operatorname{dist}_{s e}(x, y)=\operatorname{dist}_{e}(x, y)^{2}$.

By Combining the functions $\Gamma$, the various distance metrics dist and the cost functions, we obtain a family of protocols. For instance, the triplet $\left(\Gamma_{i d}\right.$, dist $_{h}$, cost $\left._{1}\right)$ corresponds to protocol VCap. For the other protocols, we consider the cost function cost $_{e}$, which is omitted for clarity, and we use the following abbreviated notation for each protocol: VCost for $\left(\Gamma_{i d}, d_{i s t_{h}}\right)$, VeCost for $\left(\Gamma_{i d}\right.$, dist $\left._{e}\right)$, VseCost for $\left(\Gamma_{i d}\right.$, dist $\left._{s e}\right)$, CVCCost for $\left(\Gamma_{v c v}\right.$, dist $\left._{h}\right), \mathrm{CVCeCost}$ for $\left(\Gamma_{v c v}\right.$, dist $\left._{e}\right)$ and CVCseCost for $\left(\Gamma_{v c v}\right.$, dist $\left._{s e}\right)$.

\section{Experimental Results}

To eliminate the effect of the MAC layer on our results, we use our own $\mathrm{C}$ simulator that assumes an ideal MAC layer, i.e. no interferences and no packet collisions. The simulated network can be described as follows. Nodes are randomly deployed in a $1 \times 1$ square using a Poisson Point Process (node positions are independent) with different density (or mean number of nodes per surface unit) $\lambda$. We assume that these nodes have the same transmission range, $R=0.1$, therefore, two nodes are connected by an edge if and only if their Euclidean distance is at most $R$ (assuming a Unit Disk Graph [3]). Finally, a set of landmarks (at least 2) is randomly selected from the network nodes.
We run the simulator using the routing algorithms described in Section 2 for the same samples of node distribution and study their performance under the family of protocols described in Section 3. The performance measures of interest in this work are the hit rate and the energy consumption. We also investigate the effect of the number of landmarks deployed on the behavior of the protocols.

- Hit rate. We define the success or hit rate as the proportion of time the routing is successful (a packet actually reaches its final destination). We compute the success rate achieved by all the cost over progress based routing protocols and the results obtained are found to be within a $95 \%$ confidence interval.
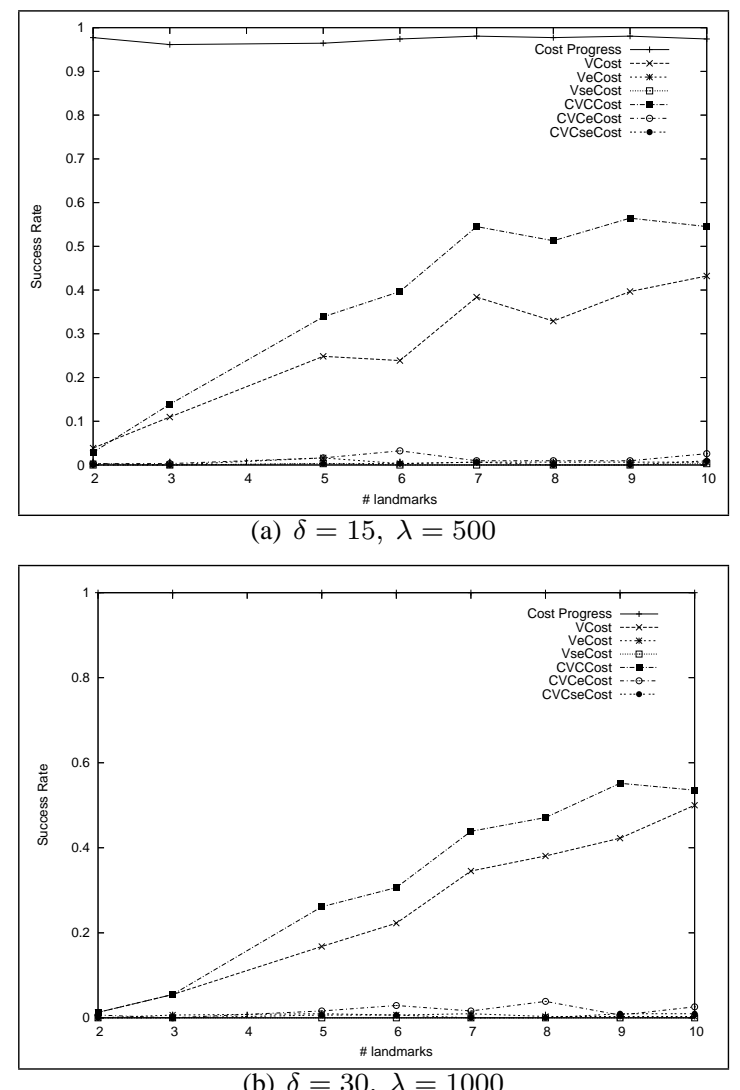

Figure 1. Hit rate computed for different coordinate systems, distance evaluations and network density $\lambda . \delta$ is the average number of neighbors per node.

In Figure 1, we plot the hit rate for two different values of $\lambda$. Clearly, the protocols using dist $t_{h}$ as the distance function (Vcost and CVCCost) achieve a better hit rate than the alternatives, independently of the node distribution density $\lambda$. Therefore, we focus on these protocols and compare them to protocols using geographic 
distance (MFR and Cost-Progress Routing protocols) and to VCap (MFR using Hamming distance over hop count coordinates).

As Figures 2(a) and 2(b) show, our protocols achieve the same hit rate as VCap. Note that our results show that VCap achieves a lower hit rate than the results published in [2]. The reason for this difference is that our work is based on a more general framework $i$. $e$., we randomly select the landmarks from the network nodes compared to the more unrealistic scenario of VCap where the landmarks are positioned on a circle around the network nodes.

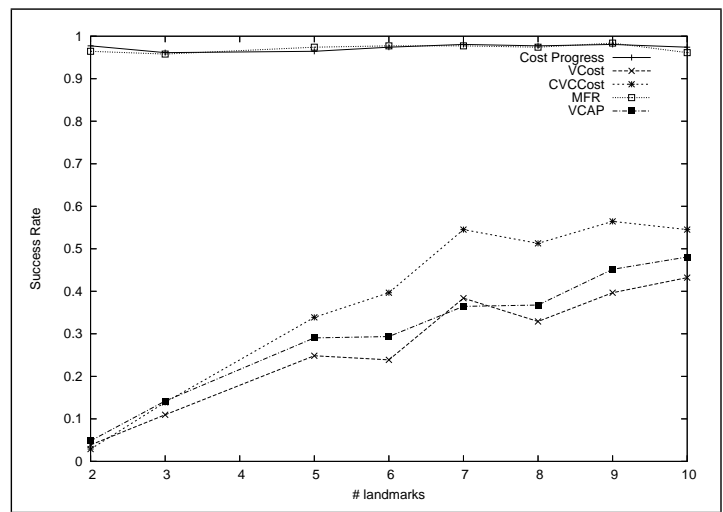

(a) Hit rate for $\delta=15, \lambda=500$

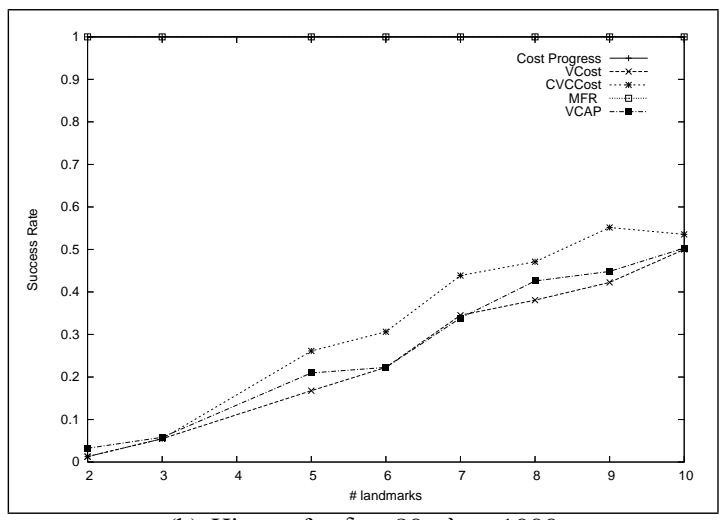

(b) Hit rate for $\delta=30, \lambda=1000$

Figure 2. Hit rate of the family of protocols using dist $_{h}$.

Figure 2 also shows that for high values of $\lambda$, the hit rate is lower than for low values of $\lambda$ which is not expected (dense networks should provide higher rate of routing success). However, our simulation results show that dense networks suffer from the redundant coordinate dilemma (high percentage of nodes with the same virtual coordinates), as Figure 3 shows. Clearly, as the number of landmarks increases, the number of nodes having the same coordinates decreases. This fact contributes to the low hit rate as we illustrate by the following example. Consider the source node $A$

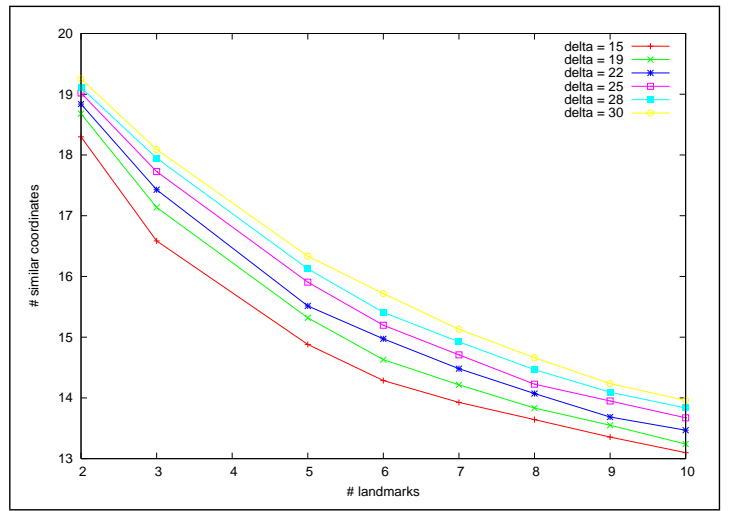

Figure 3. Number of nodes that have the same virtual coordinates computed for the family of protocols for various network density $\lambda$.

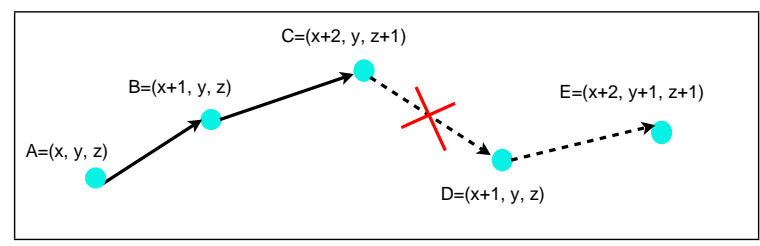

Figure 4. Illustration of failed routing due to the presence of nodes with the same virtual coordinates in the network.

in Figure 4, that is trying to send a packet to destination node $E$. Assuming a greedy routing policy, node $A$ successfully sends the packet to node $B$ since it is closer than itself to $E$; for the same reason, $B$ sends the packet to node $C$. However, $C$ does not send it to its (only) neighbor $D$, which has the same virtual coordinates as node $B$, since $C$ is closer to $E$ than $D$. Thus the routing fails and the packet never reaches its final destination.

- Energy consumption. To compare the performance of the various protocols in terms of energy efficiency, we compute the energy consumption only for successful routing paths. Figure 5 shows that VCost outperforms all the routing protocols that use dist $_{h}$ as a distance measure, CVCCost and MFR being the worst performing protocols. This conclusion is independent of the network density and the number of landmarks deployed (see Figures 5(a) and 5(b)).

To further understand this result, we compute for each protocol the routing path from a given source to a given destination (see Figure 6). Our results show that VCap 


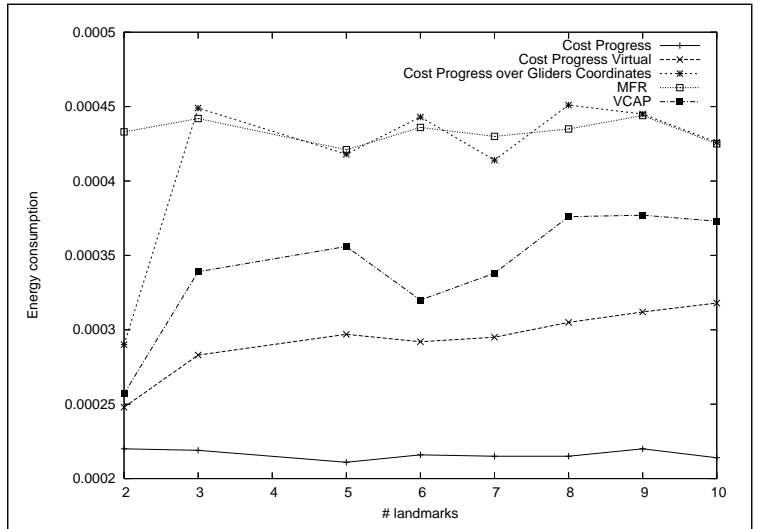

(a) Energy consumption for $\delta=15, \lambda=500$

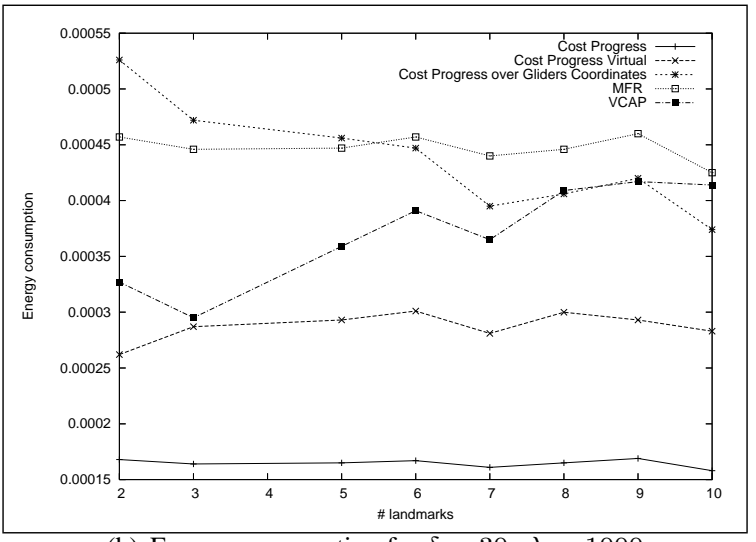

(b) Energy consumption for $\delta=30, \lambda=1000$

Figure 5. Energy consumption of the family of protocols using dist . $_{\text {. }}$

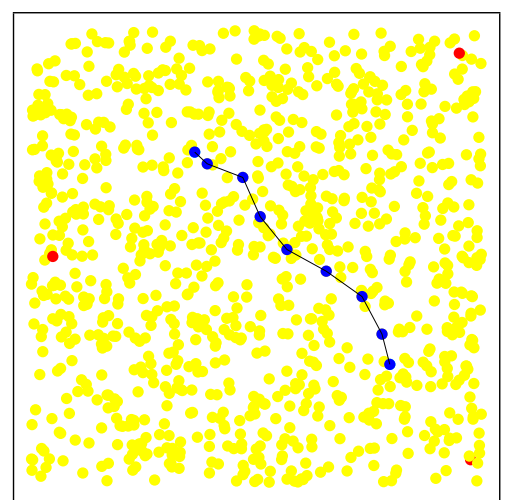

(a) MFR Path

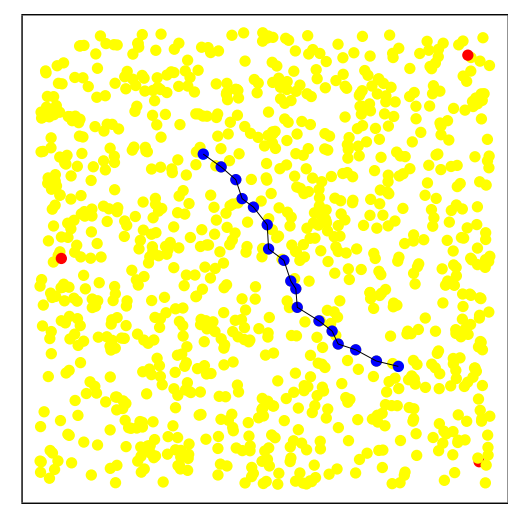

(b) Cost-Progress Path

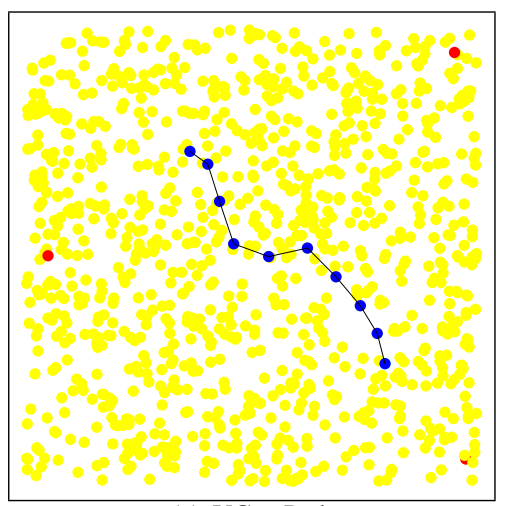

(c) VCap Path

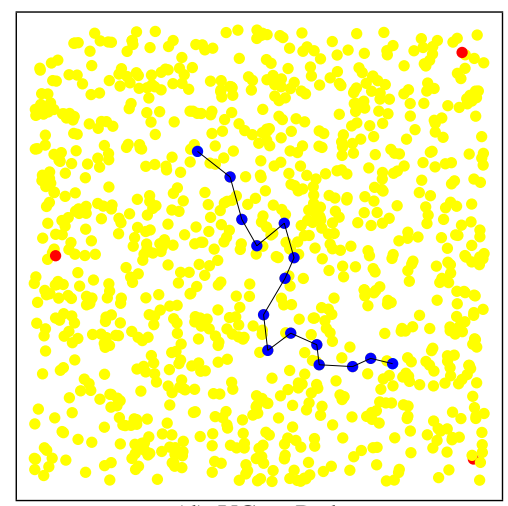

(d) VCost Path

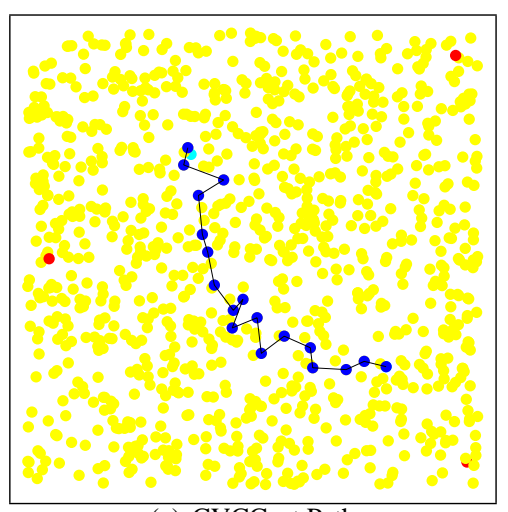

(e) CVCCost Path

Figure 6. Path followed between a pair of nodes by each protocol when $\lambda=1000$. Landmarks are shown in red. In plots (a) and (b) we use geographic coordinates and in plots (c), (d) and (e) we use virtual coordinates. 
and MFR take long edges in order to move as close as possible to the destination while Cost-Progress based routing and VCost try to minimize their energy consumption by following edges with length as close as possible to the optimal length. CVCCost on the other hand, follows very short and long edges in its route. It also has the tendency to follow paths with high number of hops compared to the other protocols. This fact makes it the worst protocol in terms of energy savings. These results are consistent for various values of network density $\lambda$ and different source/destination pairs.

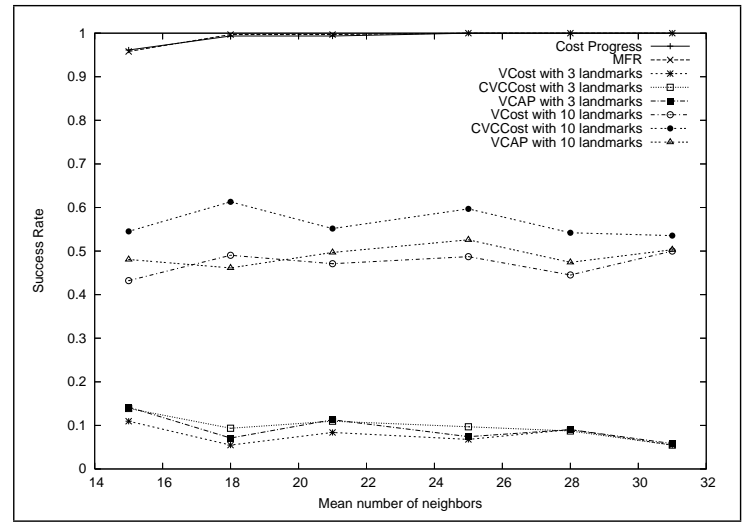

(a) Hit Rate

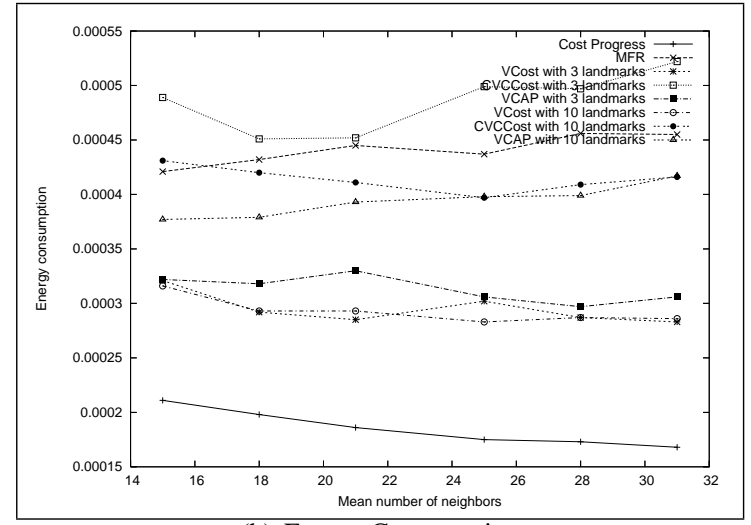

(b) Energy Consumption

Figure 7. Hit rate and energy consumption of the family of protocols as a function of the average node degree, for 3 and 10 landmarks.

- Effect of the number of landmarks on the performance measures. Figure 7 shows that for various values of $\lambda$, as the number of landmarks increases, the performance measures achieve better results. This is obviously due to the fact that adding landmarks improves the estimation of the virtual coordinates, as claimed in [1], and increases the percentage of unique virtual coordinates in the network. A drawback of increasing the number of landmarks however, is the increase of the prerouting complexity since the larger the number of deployed landmarks, the higher the dimensionality of the coordinates. Moreover, the routing protocol ends up with more paths to compute toward the landmarks in order to determine the virtual coordinates.

Figure 7 shows that our protocols achieve the best results compared to the other protocols independently of the number of landmarks.

\section{Conclusion and Future Work}

In this paper, we show how to introduce energy efficiency in position-based routing over virtual coordinates. We compare several routing protocols based on different methods to compute the virtual coordinates. We show through simulation that our protocol VCost improves significantly energy consumption and preserves the small percentage of successful routings. In our future work, we plan to test our protocol on more general networks $(i$. $e$., networks with obstacles) and to study the computation of virtual coordinates in order to increase both the success rate and the energy savings. Another interesting problem to consider is self-organization and election of landmarks.

\section{References}

[1] F. Benbadis, J.-J. Puig, M. D. de Amorim, C. Chaudet, T. Friedman, and D. Simplot-Ryl. Jumps: Enhanced hopcount positioning in sensor networks using multiple coordinates. submitted to Elsevier, 2007.

[2] A. Caruso, S. Chessa, S. De, and A. Urpi. GPS free coordinate assignment and routing in wireless sensor networks. INFOCOM 2005. Proc. IEEE., 1:150-160, 2005.

[3] B. N. Clark, C. J. Colbourn, and D. S. Johnson. Unit disk graphs. Discrete Math., 86(1-3):165-177, 1990.

[4] Q. Fang, J. Gao, L. Guibas, V. de Silva, and L. Zhang. Glider: gradient landmark-based distributed routing for sensor networks. INFOCOM 2005. Proc. IEEE., 1:339-350, 2005.

[5] J. Kuruvila, A. Nayak, and I. Stojmenovic. Progress and location based localized power aware routing for ad hoc sensor wireless networks. Int. Journal of Distrib. Sensor Networks, 2:147-159, 2006.

[6] D. Niculescu and B. Nath. Ad hoc positioning system (APS). In Proceedings of GLOBECOM, San Antonio, 2001.

[7] D. Niculescu and B. Nath. Dv based positioning in ad hoc networks. Journal of Telecommunication Systems, 2003., 22(14):267-280, 2003.

[8] I. Stojmenovic and X. Lin. Power-aware localized routing in wireless networks. IEEE Trans. Parallel Distrib. Syst., 12(11):1122-1133, 2001.

[9] H. Takagi and L. Kleinrock. Optimal tarnsmission ranges for randomly distributed packet radio terminals. IEEE transaction on communications, com-22(3):246-257, 1984. 